\title{
Quantum discord and quantum phase transition in spin-1/2 frustrated Heisenberg chain
}

\author{
Chu-Hui Fan, ${ }^{1}$ Heng-Na Xiong, ${ }^{2}$ Yixiao Huang, ${ }^{3}$ and Zhe Sun ${ }^{1, *}$ \\ ${ }^{1}$ Department of Physics, Hangzhou Normal University, Hangzhou 310036, China \\ ${ }^{2}$ Department of Physics and Center for Quantum Information Science, \\ National Cheng Kung University, Tainan 70101, Taiwan \\ ${ }^{3}$ Zhejiang Institute of Modern Physics, Department of Physics, Zhejiang University, Hangzhou 310027, China
}

(Dated: June 15, 2021)

\begin{abstract}
By using the concept of the quantum discord (QD), we study the spin- $1 / 2$ antiferromagnetic Heisenberg chain with next-nearest-neighbor interaction. Due to the $S U(2)$ symmetry and $Z_{2}$ symmetry in this system, we obtain the analytical result of the QD and its geometric measure (GMQD), which is determined by the two-site correlators. For the 4-site and 6-site cases, the connection between GMQD (QD) and the eigenenergies was revealed. From the analytical and numerical results, we find GMQD (QD) is an effective tool in detecting both the first-order and the infinite-order quantum-phase-transition points for the finite-size systems. Moreover, by using the entanglement excitation energy and a universal frustration measure we consider the frustration properties of the system and find a nonlinear dependence of the GMQD on the frustration.
\end{abstract}

PACS numbers: 03.67.-a, 64.70.Tg, 75.10.Jm

\section{INTRODUCTION}

Quantum correlation is one of the most popular research topics since it plays a central role in quantum information and communication. Usually people thought that the quantum computation devices should get their computational power from entanglement-one of the most essential nonclassical features in quantum mechanics. Recently, it was found that there exist other nonclassical relations apart from quantum entanglement. The concept of the quantum discord (QD) which is defined as the difference between the quantum mutual information and the classical correlation was introduced by Ollivier and Zurek [1] to quantify the non-classical correlations. It has been observed that the QD provided a larger region of quantum states with non-classical correlations, for example, even some separable states have non-zero QD $[2,3]$. In fact, only zero discord is a necessary condition for strictly classical correlations [4], so that the states with non-zero QD are responsible for the efficiency of a quantum computer [5]. Therefore, QD could be a new resource for quantum computation, and even far cheaper and easier to maintain in the lab.

Quite recently, many people devoted into the study of quantum discord [6-19]. Although there are several advantages, a big problem for the application of discord is that it is complicated to calculate QD analytically. Even for the two-qubit systems, the analytic results of QD can only be obtained for a few cases such as $X$-type states [20], and a general method still lacks. Since that, Dakić et al. [21] study the necessary and sufficient condition for the existence of non-zero QD for bipartite states and introduce a geometric measure of quantum discord

*Electronic address: sunzhe@hznu.edu.cn
(GMQD), which can be evaluated for an arbitrary twoqubit state. The concept of the GMQD simplifies the calculation of the QD.

In quantum many-body correlated system, quantum phase transition (QPT) is an essential phenomenon and attracts widespread attention. As a quantum critical phenomenon, QPT happens at absolute zero temperature, at which the thermal fluctuations vanish so that no classical phase transition could occur. Thus QPT is driven only by quantum fluctuation and caused by changing the system's Hamiltonian, such as an external magnetic field or the coupling constant. At the quantum phase transition point, the QPT behaves as the configuration transition of the ground state (GS). Therefore, one can easily expect that some quantum concepts closely related to the ground states can be used to indicate QPTs. For example, the concepts of quantum entanglement [2227], quantum fidelity [28-33] and quantum squeezing [34], have already been widely and successfully employed to study QPTs.

As a concept of quantum correlation, it is natural to consider the relation between the QD and the QPTs. Recently, people become to make use of QD to investigate QPTs. In Ref. [35], it shows that QD spotlights the QPT point for $X X Z$ Heisenberg chain even at finite temperature. On the other hand, the quantum criticalities in the environment also play an important role in the dynamics of the QD $[36,37]$. In some typical systems with QPTs, it is found that both classical correlation and quantum discord exhibit signatures of the QPTs [38]. All these works imply that QD is an effective tool in detecting QPTs.

In this paper, in terms of the concept of QD, we will consider the QPTs of the spin- $1 / 2$ Heisenberg chain with next-nearest-neighbor interaction which is also called the $J_{1^{-}} J_{2}$ model. This is an interesting quantum many-body system for the existence of competition between nearestneighbor $(\mathrm{NN})$ and next-nearest-neighbor $(\mathrm{NNN})$ cou- 
plings. It well describes the material structure in some quasi-one-dimensional compounds, such as $\mathrm{CuGeO}_{3}$ [39]. There are two important QPTs in this model $[40,41]$, a first-order QPT and an infinite-order QPT. The firstorder QPT lies on the energy-level crossing of the ground state (GS), while the infinite-order QPT is found to be closely related to the energy-level crossing of the lowlying excited states (ESs) and can be detected by the first-ES fidelity [33]. However, the properties of the QD in this system is lack of study, which motivates us to consider and show that the QD of the GS and the ESs is effective to indicate the QPTs in this system.

This paper is organized as follows: in Sec. II, we introduce the conception of the QD and the GMQD. In Sec. III, we give a general analytical result of the GMQD for the $X$-type states. In Sec.IV, we analytically calculate the eigenenergies of the $J_{1^{-}} J_{2}$ model for the 4-site and 6-site cases, then obtain the two-site GMQD and QD analytically. In Sec. V, we will numerically show that the GMQD is a good candidate to indicate the QPTs. In Sec. VI, we consider the frustration by making use of the entanglement excitation energy (EXE) and a universal measure $f$. Then we find the relation between frustration and the GMQD. Finally, the conclusion will be given.

\section{QUANTUM DISCORD AND ITS GEOMETRIC MEASURE}

\section{A. Quantum discord}

Given a quantum state $\rho$ in a composite Hilbert space $\mathcal{H}=\mathcal{H}_{A} \otimes \mathcal{H}_{B}$, the total amount of correlation is quantified by quantum mutual information

$$
\mathcal{I}(\rho)=S\left(\rho_{A}\right)+S\left(\rho_{B}\right)-S(\rho)
$$

where $S(\rho) \equiv-\operatorname{Tr}\left[\rho \log _{2} \rho\right]$ is the von Neumann entropy and $\rho_{A(B)}=\operatorname{Tr}_{B(A)} \rho$ is the reduced density matrix by tracing out system $B(A)$. If we take the system $A$ as the apparatus, the quantum discord is defined as follows $[1$, $42]$

$$
D(\rho)=\mathcal{I}(\rho)-\mathcal{C}_{A}(\rho),
$$

which is the difference of the total amount of correlation $\mathcal{I}(\rho)$ and the classical correlation $\mathcal{C}_{A}(\rho)$. Here the classical correlation is defined by

$$
\mathcal{C}(\rho)=\max _{\left\{E_{k}\right\}} \mathcal{I}\left(\rho \mid\left\{E_{k}\right\}\right),
$$

where $\mathcal{I}\left(\rho \mid\left\{E_{k}\right\}\right)$ is a variant of quantum mutual information based on a given measurement basis $\left\{E_{k}\right\}$ on system $A$ as follows

$$
\mathcal{I}\left(\rho \mid\left\{E_{k}\right\}\right)=S\left(\rho_{B}\right)-\sum_{k} p_{k} S\left(\rho_{B \mid k}\right)
$$

$\rho_{B \mid k}=\operatorname{Tr}_{A}\left[\left(E_{k} \otimes \mathbb{I}\right) \rho\right] / p_{k}$ is the postmeasurement state of $B$ after obtaining outcome $k$ on $A$ with the probability $p_{k}=\operatorname{Tr}\left[\left(E_{k} \otimes \mathbb{I}\right) \rho\right] . \quad\left\{E_{k}\right\}$ is a set of one-dimensional projectors on $\mathcal{H}_{A}$, and $\mathbb{I}$ is the $2 \times 2$ identity operator.

\section{B. Geometric measure of the quantum discord}

In Ref. [21], Dakić et al. proposed a geometric measure of quantum discord defined by

$$
D_{g}(\rho):=\min _{\chi \in \Omega_{0}}\|\rho-\chi\|^{2},
$$

where $\Omega_{0}$ denotes the set of zero-discord states and $\|X\|^{2}:=\operatorname{Tr}\left(X^{\dagger} X\right)$ is the Hilbert-Schmidt norm. For two-qubit systems, a general state can be written in the Bloch representation [21, 43]:

$$
\rho=\frac{1}{4} \mathbb{I} \otimes \mathbb{I}+\sum_{i}^{3}\left(x_{i} \sigma_{i} \otimes \mathbb{I}+y_{i} \mathbb{I} \otimes \sigma_{i}\right)+\sum_{i, j=1}^{3} R_{i j} \sigma_{i} \otimes \sigma_{j}
$$

with the real parameters $x_{i}, y_{i}$, and $R_{i j}$, and the Pauli matrices $\sigma_{i=1,2,3}$. Here we only consider the case that the measurement is taken on the system $A$. Then an explicit expression of the GMQD is obtained as [21]:

$$
D_{g}(\rho)=\frac{1}{4}\left(\|x\|^{2}+\|R\|^{2}-k_{\max }\right)
$$

where $x=\left(x_{1}, x_{2}, x_{3}\right)^{T}, R$ is the matrix with elements $R_{i j}$, and $k_{\max }$ is the largest eigenvalue of matrix $K=$ $x x^{T}+R R^{T}$.

Here we introduce an alternative form which will be convenient when we consider the evolution of the GMQD under decoherence. First, we introduce a matrix $\mathcal{R}$ defined by [44]

$$
\mathcal{R}=\left(\begin{array}{cc}
1 & y^{T} \\
x & R
\end{array}\right)
$$

and another $3 \times 4$ matric $\mathcal{R}^{\prime}$ obtained through deleting the first row of $R$ :

$$
\mathcal{R}^{\prime}=(x, R)
$$

Here $\mathcal{R}$ is just an expectation matrix with the elements $\mathcal{R}_{i j}=\operatorname{Tr}\left[\rho \sigma_{i} \otimes \sigma_{j}\right]$ for $i, j=0,1,2,3$, and $\sigma_{0}=\mathbb{I}$ is defined. The expression (9) leads to $K=\mathcal{R}^{\prime}\left(\mathcal{R}^{\prime}\right)^{T}$. After singular value decomposition, we have $\mathcal{R}^{\prime}=U \Lambda V^{T}$, where $U$ and $V$ are $3 \times 3$ and $4 \times 4$ orthogonal matrices, and $\Lambda$ has only diagonal elements $\Lambda_{i j}=\mu_{i} \delta_{i j}$ with $\mu_{i}$ the so-called singular values of the matrix $\mathcal{R}^{\prime}$. Then the eigenvalues of the matrix $K$ can be expressed as $\mu_{i}^{2}$. Considering $\|x\|^{2}+\|R\|^{2}=\operatorname{Tr} K$, we get an alternative compact form of $D_{g}(\rho)$ :

$$
D_{g}(\rho)=\frac{1}{4}\left[\left(\sum_{k} \mu_{k}^{2}\right)-\max _{k} \mu_{k}^{2}\right]
$$


where the summation and maximization arı all the non-zero singular values $\mu_{k}$ of $\mathcal{R}^{\prime}$. Thi form will be convenient when we calculate th the $J_{1}-J_{2}$ model.

\section{The Geometric measure of the quant for $X$-state}

In the standard basis of operator $S_{z}=s_{1}$ density matrix of the so-called " $X$-state" is s lows:

$$
\rho=\left(\begin{array}{cccc}
a & 0 & 0 & g \\
0 & b & w & 0 \\
0 & w^{*} & c & 0 \\
g^{*} & 0 & 0 & d
\end{array}\right)
$$

where the parameters $a, b, c$ and $d$ are real numbers and satisfy $a+b+c+d=1$, and the positive condition requires $b c \geq|w|^{2}$ and $a d \geq|g|^{2}$. Then we can obtain parameter matrix $R$ as:

$$
R=\left(\begin{array}{ccc}
R_{11} & R_{12} & 0 \\
R_{21} & R_{22} & 0 \\
0 & 0 & R_{33}
\end{array}\right)
$$

where $R_{11}=2 \operatorname{Re}(g+w), R_{12}=2 \operatorname{Im}(w-g), R_{21}=$ $-2 \operatorname{Im}(g+w), R_{22}=2 \operatorname{Re}(w-g)$, and $R_{33}=a-b-c+d$. The parameter vectors $x=\left(0,0, x_{3}\right)^{T}$ with $x_{3}=a+b-$ $c-d$, and the vector $y=\left(0,0, y_{3}\right)^{T}$ with $y_{3}=a-b+c-d$. By substituting the elements of $R$ and $x$ into the matrix $\mathcal{R}^{\prime}=(x, R)$ (Eq. (9)), we can calculate the GMQD by using Eq. (10) that

$$
D_{g}(\rho)=\frac{1}{4}\left[\mu_{1}^{2}+\mu_{2}^{2}+\mu_{3}^{2}-\max \left(\mu_{1}^{2}, \mu_{3}^{2}\right)\right],
$$

where $\mu_{1}^{2}=4(|g|+|w|)^{2}, \mu_{2}^{2}=4(|g|-|w|)^{2}$ and $\mu_{3}^{2}=$ $2\left[(a-c)^{2}+(b-d)^{2}\right]$. The expression above is the analytical result of the GMQD for the $X$-states, which represent an important class of quantum states, e.g., the $S U(2)$-symmetric states.

\section{GMQD FOR THE HEISENBERG MODEL WITH NNN INTERACTIONS}

The Hamiltonian of one-dimensional Heisenberg system with NNN interaction, i.e., the $J_{1^{-}} J_{2}$ model reads (let $\hbar=1$ )

$$
H=\sum_{i}^{N}\left(J_{1} \mathbf{s}_{i} \cdot \mathbf{s}_{i+1}+J_{2} \mathbf{s}_{i} \cdot \mathbf{s}_{i+2}\right),
$$

where the $\mathbf{s}_{i}$ denotes the spin- $1 / 2$ operator at the $i_{\text {th }}$ site. $N$ is the total number of sites and here we only consider the even case. $J_{1}$ and $J_{2}$ are the nearest-neighbor
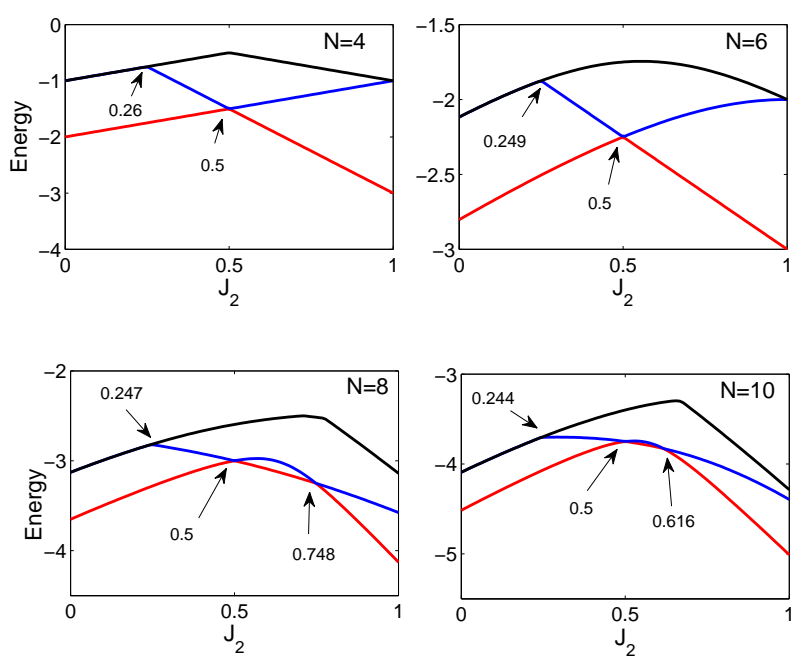

FIG. 1: Low-level energy spectrum of the spin-1/2 Heisenberg chain with NNN interaction for different system sizes $N=$ $4,6,8,10$. The parameters are in units of $J_{1}$.

(NN) and next-nearest-neighbor (NNN) exchange couplings. As usual, we choose the periodic boundary condition. Note that no exact analytical results are available for this model (14) except for the special case of $J_{2} / J_{1}=0$ and $J_{2} / J_{1}=1 / 2$. There are two important QPTs in this model, a first-order QPT at $J_{c 1}=0.5$ and an infinite-order QPT at $J_{c 2} \simeq 0.241$ (where $J_{c}=J_{2} / J_{1}$ ).

At the critical point $J_{c 1}=0.5$, the system reduces to the Majumdar-Ghosh model [40]. Its GS is of spin zero, but degenerate, which is a uniformly weighted superposition of the singlet states between NN sites (for even and infinite $\mathrm{N}$ cases). This point is just the GS energy-level crossing induced by the translation symmetry breaking, thus it is a first-order QPT point.

At the other critical point $J_{c 2} \simeq 0.241$, the system undergoes a Berezinskii-Kosterlitz-Thouless (BKT) type QPT from spin fluid to dimerized phase [41]. This phase transition is driven by the competition between the NN and the NNN interactions. When $J_{2} / J_{1}<J_{c 2}$, the NNN interaction does not change the character of the simple antiferromagnetic case $J_{2} / J_{1}=0$, whose GS is described as spin fluid massless spinon excitations. When $J_{2} / J_{1}>$ $J_{c 2}$, the frustration term is relevant and the GS flows to the strong-coupling dimerized phase. Furthermore, it is found that $J_{c 2}$ is accurately the energy-level crossing point of the first ESs for even-size and infinite-size cases.

As shown in figure 1, we plot the low-energy levels of the GS and the first and second ES versus different coupling $J_{2}$ (let $J_{1}=1$ ). Clearly, for different sizes of $N=4,6,8,10$, the GS energy-level crossing occurs at the point $J_{c 1}=0.5$, which exactly corresponds to the first-order QPT point. Turning to the energy levels of the first ES, we find that with the increasing system size, the crossing position moves from 0.25 to 0.244 , which implies that in the thermodynamic limit, the energy-level 
crossing point of the first ES will tend to the BKT QPT point $J_{c 2} \simeq 0.241$. From the numerical results above, we emphasize that the QPTs are closely related to the fundamental change of the energy structure. Consequently, the corresponding eigenstates will present special quantum properties at the QPT points.

Chen et al [33] studied the ground-state fidelity and first-excited-state fidelity of this system. Xiong et al [29] found the reduced fidelity can also be used to indicate the quantum criticalities. In this paper, we will show that the two-site QD of the GS and the first ES is also effective to detect the QPTs.

Due to the $S U(2)$ symmetry and the $Z_{2}$ symmetry generated by commutation $\left[\sigma_{x, y, z}^{\otimes^{N}}, H\right]=0$, the reduced density matrix for the two-site subsystem $\rho_{i j}=\operatorname{Tr}_{\overline{i j}}\left(\rho_{\text {tot }}\right)$ with $\operatorname{Tr}_{\overline{i j}}$ meaning tracing out the subsystems except the $i$-th and $j$-th ones:

$$
\rho_{i j}=\left(\begin{array}{cccc}
a & 0 & 0 & 0 \\
0 & b & w & 0 \\
0 & w & b & 0 \\
0 & 0 & 0 & a
\end{array}\right),
$$

where all the elements are real numbers. Note that if the states are degenerate, the reduced density matrices may not be in the form above. For example, for the evensize $J_{1}-J_{2}$ Heisenberg system, the first ES is threefold degenerate when $0<J_{2} / J_{1}<J_{c 2}\left(J_{c 2} \simeq 0.241\right)$, and is nondegenerate when $J_{2} / J_{1}>J_{c 2}$ except for the crossing points. In order to overcome the above subtle problem induced by the degeneracy, we mix the degenerate states with equal probability as that

$$
\rho_{n}=\frac{1}{G} \sum_{v=1}^{G}\left|\psi_{n v}\right\rangle\left\langle\psi_{n v}\right|,
$$

where $G$ denotes the degeneracy of the energy $E_{n}$ and $\left|\psi_{n v}\right\rangle$ the $v$-th degenerate eigenstate of the system. This assumption is reasonable when we consider a general mixed state in the thermal equilibrium $\rho(T)=$ $\exp \left[-H /\left(k_{b} T\right)\right] / Z$, with $Z$ is the partition funciton. If the eigenstate $\left|\psi_{n}\right\rangle$ of the Hamiltonian $H$ is degenerate, each of its degenerate states will have an equal mixture weight in the mixed thermal state. It is easy to prove that the equal-probability mixed state holds the $S U(2)$ and $Z_{2}$ symmetry, i.e., the reduced density is still in the form of Eq. (15).

By using the results of GMQD for $X$-state in Eq. (13), we obtain the GMQD for the reduced density matrix $\rho_{i j}$ as

$$
D_{g}\left(\rho_{i j}\right)=\frac{1}{4}\left[\mu_{1}^{2}+\mu_{2}^{2}+\mu_{3}^{2}-\max \left(\mu_{1}^{2}, \mu_{3}^{2}\right)\right],
$$

where $\mu_{1}^{2}=4(a-b)^{2}$ and $\mu_{2}^{2}=\mu_{3}^{2}=4 w^{2}$.

It is known that the elements of the reduced density matrix can be presented by the expectation values of the
Pauli matrices of the two-site subsystem, i.e., $\left\langle\sigma_{i, \alpha} \sigma_{j, \beta}\right\rangle$ (with $\alpha, \beta=x, y, z$ ). In addition, there is an exchange invariance in the Hamiltonian, which leads to the fact that, any NN-site correlators, i.e., $\left\langle\sigma_{i, \alpha} \sigma_{i+1, \beta}\right\rangle$, equal to each other, so to the NNN-site correlators $\left\langle\sigma_{i, \alpha} \sigma_{i+2, \beta}\right\rangle$. Thus when we consider the reduced density of the two-site subsystem, the elements can be described by the correlators

$$
\begin{aligned}
a & =\frac{1}{4}\left(1+\left\langle\sigma_{i z} \sigma_{j z}\right\rangle\right), \\
b & =\frac{1}{4}\left(1-\left\langle\sigma_{i z} \sigma_{j z}\right\rangle\right), \\
w & =\frac{1}{4}\left(\left\langle\sigma_{i x} \sigma_{j x}\right\rangle+\left\langle\sigma_{i y} \sigma_{j y}\right\rangle\right),
\end{aligned}
$$

where $\sigma_{x, y, z}$ are Pauli matrices. In this system, there exists another relation that $\left\langle\sigma_{i \alpha} \sigma_{j \alpha}\right\rangle=\frac{1}{3}\left\langle\sigma_{i} \sigma_{j}\right\rangle$ with $\alpha=$ $x, y, z$ and $\sigma_{i} \sigma_{j}=\sigma_{i x} \sigma_{j x}+\sigma_{i y} \sigma_{j y}+\sigma_{i z} \sigma_{j z}$. Therefore, we can prove that the three values $\mu_{1,2,3}$ in Eq. (17) equal to each other. Then the GMQD becomes

$$
D_{g}\left(\rho_{i j}\right)=\frac{1}{2}\left\langle\sigma_{i \alpha} \sigma_{j \alpha}\right\rangle^{2}=\frac{1}{18}\left\langle\sigma_{i} \sigma_{j}\right\rangle^{2} .
$$

This equation is a key result of this paper, and gives a general result for the Heisenberg systems which contains the same symmetry properties of the $J_{1}-J_{2}$ model, such as $X X X$-type and dimerized Heisenberg chain.

Furthermore, for the $J_{1}-J_{2}$ model, by using of the Feynman-Hellman theorem, one can find the correlators between the $\mathrm{NN}$ sites is

$$
\left\langle\sigma_{i} \sigma_{i+1}\right\rangle=\frac{4}{J_{1}}\left[e-J_{2} \frac{\partial e}{\partial J_{2}}\right],
$$

where $e=\langle H\rangle / N$ denotes the expectation value of the energy corresponding to each site. Similarly, for the NNN sites, we can obtain the correlator $\left\langle\sigma_{i} \sigma_{i+2}\right\rangle=4 \partial e / \partial J_{2}$. Finally the NN-site GMQD will be connected to the energy that

$$
D_{g}\left(\rho_{i, i+1}\right)=\frac{8}{9 J_{1}^{2}}\left(e-J_{2} \frac{\partial e}{\partial J_{2}}\right)^{2},
$$

and the NNN-site GMQD is

$$
D_{g}\left(\rho_{i, i+2}\right)=\frac{1}{18}\left\langle\sigma_{i} \sigma_{j}\right\rangle^{2}=\frac{8}{9} \frac{\partial e}{\partial J_{2}} .
$$

The two equation above present the connection between the GMQD and the energy. We can also give the QD of this model by using the results of [16]

$$
\begin{aligned}
D\left(\rho_{i j}\right)= & -2(a+b) \log _{2}(a+b)+2 a \log _{2} a+2 b \log _{2} b \\
& +2 w \log _{2} w-\frac{1}{2}\left[\left(1+4 w^{2}\right) \log _{2}\left(1+4 w^{2}\right)\right. \\
& \left.+\left(1-4 w^{2}\right) \log _{2}\left(1-4 w^{2}\right)\right],
\end{aligned}
$$

by substituting the Eqs. (18) and (20), one can find the relation between the QD and the energy. 
From the Eq. (21), we find that the GMQD is dominated by the energy structure and its derivative. Obviously, the GMQD will be sensitive to the singularity of the energy level. In other words, the GMQD can effectively indicate the energy crossings. As is known, the first-order QPTs usually lies on the energy-level crossing of the GS. Hence, we can reasonably expect that the GMQD can indicate the first-order QPTs effectively. In the $J_{1}-J_{2}$ model, there is another QPT point nearby $J_{c 2} \simeq 0.241$, which corresponds to the energy-level crossing of the first ESs. Therefore, we can use the GMQD of the first ES to detect the second QPT point $J_{c 2}$.

In the following sections, we will analytically and numerically calculate the small-size cases, which can also justify the fact that the GMQD can indicate the QPTs in the $J_{1}-J_{2}$ model.

\section{ANALYTICAL RESULTS FOR THE CASES OF $N=4$ AND $N=6$}

In this section, we will analytically calculate the $J_{1}$ $J_{2}$ model for 4 -site and 6 -site cases. The eigenenergy structure can be directly used to obtain the GMQD. In the following, we set $J_{1}=1$ for simplicity.

Due to the periodic boundary condition, the Hamiltonian is translational invariant, i.e., $[H, T]=0$, where $T$ defines the cyclic right-shift operator satisfying $T\left|m_{1} m_{2} \ldots m_{i-1} m_{i}\right\rangle=\left|m_{i} m_{1} \ldots m_{i-1}\right\rangle$.

\section{A. 4-site case}

For $\left[H, J_{z}\right]=0$, the 16-dimensional Hilbert space for a 4 -site $J_{1}-J_{2}$ model can be divided into invariant subspaces spanned by vectors with a fixed number of reversed spins [45]. Thus, the dimension of the largest subspace is 6 . With the help of translational invariance, we can further reduce the Hamiltonian matrix to $2 \times 2$ submatrices, and then the eigenvalue of Hamiltonian can be solved.

The none-reversed subspace contains only one vector $|0000\rangle$, which the eigenvalue is $E=1$. The subspace with one reversed is spanned by 4 basis vectors, $\left\{T^{n}|1000\rangle, n=0,1,2,3\right\}$. Considering the translational invariance of the Hamiltonian, we choose another set of basis vectors [46]:

$$
\left|\Psi_{k}\right\rangle=\sum_{n=0}^{3} \omega_{k}^{n} T^{n}|1000\rangle,
$$

where $\omega_{k}=e^{i 2 k \pi / 4}, k \in\{0,1,2,3\}$. One can check that $\left|\Psi_{k}\right\rangle$ is eigenstates of $T$ with eigenvalues $\omega_{k}^{-1}$, and also are eigenstates for $H$ with eigenvalues $\frac{1}{2}\left(-1+\omega_{k}^{-1}+J_{2} \omega_{k}^{-2}+\right.$ $\left.\omega_{k}^{-3}\right)$.

When the reversed number is 2 or 3 , we choose the basis

$$
\left|\Psi_{k}\right\rangle=\sum_{n=0}^{3} \omega_{k}^{n} T^{n}|1100\rangle,
$$

and

$$
\left|\Phi_{k}\right\rangle=\sum_{n=0}^{3} \omega_{k}^{n} T^{n}|1010\rangle,
$$

for the 6-dimensional subspace. Under this space, one can rewrite the Hamiltonian into a $2 \times 2$ form and obtain all the eigenvalues. Then, the GS energy is

$$
E=\left\{\begin{array}{cc}
-2+J_{2} & \text { when } J_{2} \leq 0.5 \\
-3 J_{2} & \text { when } J_{2} \geq 0.5
\end{array}\right.
$$

Substituting the energy levels above into Eq. (21), one can obtain the NN-site GMQD corresponding to the GS $\rho_{0}$ :

$$
D_{g}\left(\rho_{0}\right)=\left\{\begin{array}{cl}
\frac{2}{9} & \text { when } J_{2} \leq 0.5 \\
0 & \text { when } J_{2} \geq 0.5 .
\end{array}\right.
$$

We also obtain the 1st ES energy:

$$
E=\left\{\begin{array}{cc}
-1+J_{2} & \text { when } J_{2} \leq 0.25 \\
-3 J_{2} & \text { when } 0.5 \geq J_{2} \geq 0.25 \\
-2+J_{2} & \text { when } J_{2} \geq 0.5
\end{array}\right.
$$

thus the NN-site GMQD corresponding to the first ES $\rho_{1 \mathrm{st}}$ :

$$
D_{g}\left(\rho_{1 \mathrm{st}}\right)=\left\{\begin{array}{cc}
\frac{1}{18} & \text { when } J_{2} \leq 0.25 \\
0 & \text { when } 0.5>J_{2} \geq 0.25 \\
\frac{2}{9} & \text { when } J_{2}>0.5
\end{array}\right.
$$

Obviously, the GMQD presents discontinuity at the point $J_{2}=0.5$, which just corresponds to the first-order QPT of this system. While for the first ES, the GMQD sudden changes at two points: $J_{2}=0.25$ and $J_{2}=0.5$. Similarly, one can obtain the NNN-site GMQD, and we do not show the results here.

\section{B. 6-site case}

For the 6 -site case, the energy structures are more complicated. Fortunately, we analytically obtain all the eigenenergies. The GS energy is:

$$
E=\left\{\begin{array}{cc}
-\frac{1}{2} \sqrt{9 J_{2}^{2}-18 J_{2}+13}-1 & \text { when } J_{2} \leq 0.5 \\
-\frac{3}{2}\left(1+J_{2}\right) & \text { when } J_{2} \geq 0.5 .
\end{array}\right.
$$

Therefore, when $J_{2} \leq 0.5$ the NN-site GMQD for the GS is 

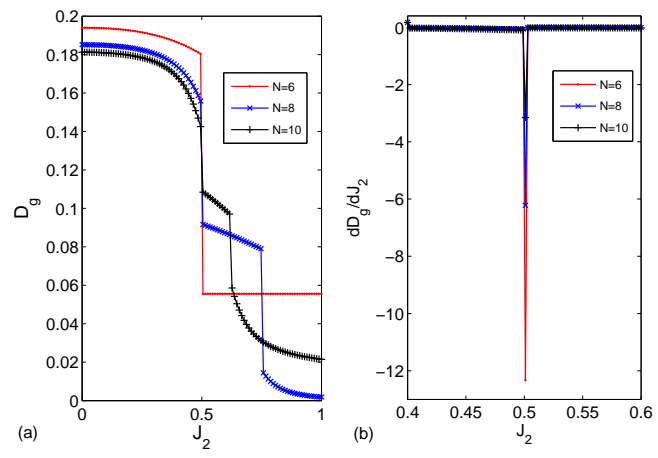
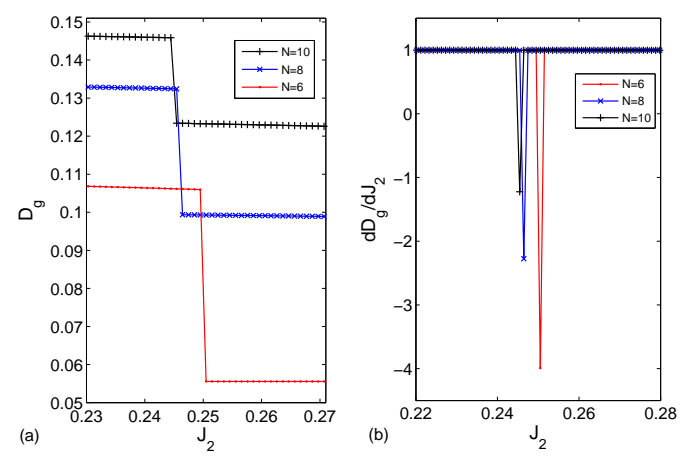

FIG. 2: (a) NN-site GMQD of the ground state versus coupling $J_{2}$ for different system sizes $N=6,8,10$; (b) The derivative of the GMQD versus $J_{2}$. The parameters are in units of $J_{1}$.

$$
D_{g}\left(\rho_{0}\right)=\frac{1}{162}\left(\frac{18 J_{2}-26}{\sqrt{9 J_{2}^{2}-18 J_{2}+13}}-1\right)^{2},
$$

and when $J_{2} \geq 0.5$, we have a constant value of the GMQD: $D_{g}\left(\rho_{0}\right)=1 / 18$.

The first ES energy is:

$E=\left\{\begin{array}{cc}-\frac{1}{2} \sqrt{9 J_{2}^{2}-10 J_{2}+5}-1 & \text { when } J_{2} \leq 0.25, \\ -\frac{3}{2}\left(1+J_{2}\right) & \text { when } 0.5 \geq J_{2} \geq 0.25, \\ -\frac{1}{2} \sqrt{9 J_{2}^{2}-18 J_{2}+13}-1 & \text { when } J_{2} \geq 0.5 .\end{array}\right.$

Thus when $J_{2} \leq 0.25$, the NN-site GMQD is

$$
D_{g}\left(\rho_{1 \mathrm{st}}\right)=\frac{2}{81}\left(\frac{9 J_{2}^{2}-19 J_{2}+10}{2 \sqrt{9 J_{2}^{2}-10 J_{2}+5}}+1\right)^{2} .
$$

When $0.5 \geq J_{2} \geq 0.25$, we have a constant value of the GMQD that $\bar{D}_{g}\left(\rho_{1 \mathrm{st}}\right)=1 / 18$. When $J_{2} \geq 0.5$, the GMQD becomes

$$
D_{g}\left(\rho_{1 \mathrm{st}}\right)=\frac{2}{81}\left(\frac{9 J_{2}^{2}-27 J_{2}+22}{2 \sqrt{9 J_{2}^{2}-18 J_{2}+13}}+1\right)^{2} .
$$

Obviously, the GMQD of the GS changes at the point $J_{2}=0.5$, and the GMQD of the ES changes at both the points $J_{2}=0.5$ and $J_{2}=0.25$.

From the analytical results of the GMQD, we see that the first-order QPT point $J_{c 1}=0.5$ can be indicated by the GMQD of the GS and ES. However, for the smallsize cases as 4 sites and 6 sites, the GMQD of the ES cannot indicate the second $\mathrm{QPT}$ point $J_{c 2} \simeq 0.241$. This is because the QPTs occurs at the thermodynamic limit
FIG. 3: (a) NN-site GMQD of the first excited states versus coupling $J_{2}$ for different system sizes $N=6,8,10$; (b)The derivative of the GMQD versus $J_{2}$. The parameters are in units of $J_{1}$.

and the critical values is obtained in the systems with infinite size. Although the small systems cannot accurately reflect the quantum criticalities, we can simulate the critical properties in the small systems [46, 47]. In the following section, we will numerically calculate the GMQD for some larger system with 8 sites and 10 sites. The second critical region can be approximately indicate by the GMQD of the ES.

\section{NUMERICAL RESULT}

In Fig. 2a, we plot the NN-site GMQD of the GS versus coupling $J_{2}$ for different system sizes $N=6,8,10$. It is seen that the GMQD presents discontinuity at the point $J_{c 1}=0.5$. This is because that the structure of the GS changes suddenly at the QPT point which can be reflected by the quantum correlation, i.e., the GMQD. In Fig. 2b, the QPT point $J_{c 1}=0.5$ is clearly detected by the derivative of the GMQD.

In order to study the BKT-type QPT, in Fig. 3a we plot the NN-site GMQD of the first ES versus coupling $J_{2}$ for different system sizes $N=6,8,10$. Obviously, there exists a sudden drop of the GMQD. Moreover, with the increasing system size, the point of the drop tends to the QPT point $J_{c 2} \simeq 0.241$. In Fig. $3 \mathrm{~b}$, it is more obviously to find the QPT point when we plot the derivative of the GMQD. The NNN-site GMQD of the first ES is shown in Fig. 4. Some different phenomena can be seen, for the case $N=6$, increasing coupling $J_{2}$ can enhance the NNN-site GMQD, and the derivative of the NNN-site GMQD presents a positive peak. With the increasing system size, the discontinuous position of the NNN-site GMQD approaches the QPT point $J_{c 2} \simeq 0.241$.

Note that as the system size increases, the discontinuous behavior around the QPT point $J_{c 1}=0.5$ and $J_{c 2} \simeq 0.241$ becomes more and more weak. One pos- 

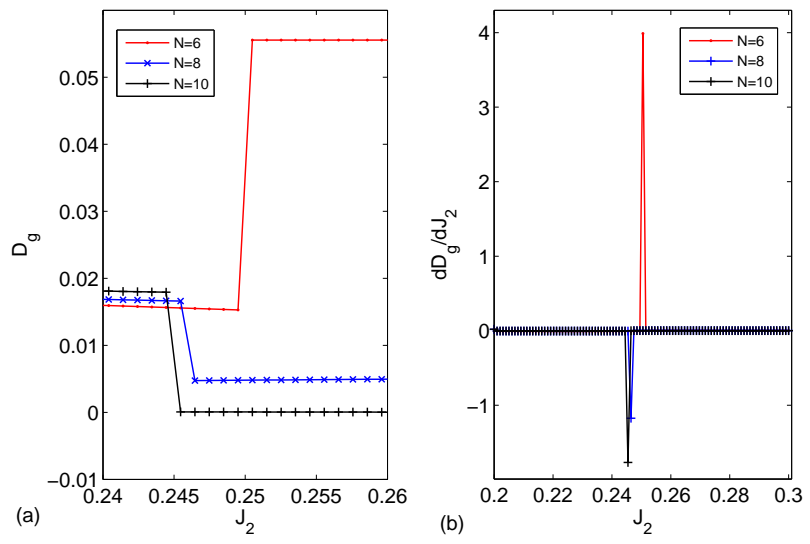

FIG. 4: (a) NNN-site GMQD of the first excited states versus coupling $J_{2}$ for different system sizes $N=6,8,10$; (b) The derivative of the GMQD versus $J_{2}$. The parameters are in units of $J_{1}$.

sible explanation is that for the QPTs associated with the energy-level crossings, the continuity of the quantum correlation characterized by the GMQD lies on the orthogonality of the eigenstates on both sides of the point. However, in this paper we only consider the GMQD in the reduced two-site subsystem where the orthogonality of the global system is destroyed. Moreover, with the increasing system size, the orthogonality, in other words, the information kept by the two-site subsystem occupies less and less proportion of the global system. Therefore, the discontinuous phenomena of the GMQD become weaker and weaker with the increasing system size. Nonetheless, this GMQD approach for small finitesize systems is still meaningful for the usual theoretical and experimental researches in the QPT problems.

We also numerically calculate the QD (defined in Eq. (2)) and find a similar discontinuous behavior with the GMQD near the QPT points, thus we do not show the numerical results of the QD in this paper. It is known that the GMQD can not absolutely characterize the QD, e.g., under a local decoherence channel, a sudden change in the decay rate of the GMQD does not always imply that of the quantum discord [44]. However in this $J_{1}-J_{2}$ model, the GMQD reflects the quantum criticalities as effectively as the QD. Furthermore, the concise expression of the GMQD is more conducive to reveal the connection between the quantum correlation and the eigenenergies. The numerical calculation of the GMQD is more timesaving than that of the QD.

\section{FRUSTRATION, GMQD AND QPT}

As is konwn that the $J_{1}-J_{2}$ model characterizes a typical frustrated spin system. Frustration arises from the simultaneous presence of competing antiferromagnetic exchange interactions of different spatial range[48], and which causes the impossibility of minimizing simultaneously the energy of competing interactions. Some methods based on quantum information techniques were introduced to investigate frustrations, e.g., the entanglement excitation energy (EXE) $\Delta E[49]$, and a universal measure of frustration $f[50]$. In this section we will analytically calculate the two quantities and find the relationship with GMQD.

The entanglement excitation energy (EXE) is defined as

$$
\Delta E=\min \left(\left\langle\Psi\left|U_{k} H U_{k}\right| \Psi\right\rangle-\langle\Psi|H| \Psi\rangle\right),
$$

where $U_{k}=\otimes_{i \neq k} I_{i} \otimes O_{k}$ with $I_{i}$ denoting the identity operator on all the spins but the one at site $k$, and $O_{k}$ is a generic Hermitian, unitary, and traceless operator can be described as $O_{k}=\sin \theta \cos \varphi \sigma_{k, x}+\sin \theta \sin \varphi \sigma_{k, y}+$ $\cos \theta \sigma_{k, z}$. If choosing the $|\Psi\rangle$ as the GS the system $H$, we know that for any translationally invariant and frustration-free Hamiltonian $H$ such that $\left[H, U_{k}\right] \neq 0$ $\forall U_{k}$, the vanishing of the EXE is a necessary and sufficient condition for GS factorization [49], and thus it is a proper measure of single-site entanglement. Indeed, the presence of frustration tends to enhance correlations among the constituents and thus to depress the possibility for the occurrence of separable (uncorrelated) states. Hence entanglement and separability can be used to qualify and quantify frustration [48].

When calculating the EXE in the $J_{1}-J_{2}$ model, due to the $S U(2)$ and $Z_{2}$ symmetry and also the translationally invariant, we have the relations $\left\langle\sigma_{i, \alpha} \sigma_{j, \beta}\right\rangle=0$ for $\alpha \neq \beta$ and $\alpha, \beta=x, y, z$, and $\left\langle\sigma_{i, x} \sigma_{j, x}\right\rangle=\left\langle\sigma_{i, y} \sigma_{j, y}\right\rangle=\left\langle\sigma_{i, z} \sigma_{j, z}\right\rangle$. Finally we obtain that

$$
\Delta E_{i}=-2 J_{1}\left\langle\sigma_{i, z} \sigma_{i+1, z}\right\rangle-2 J_{2}\left\langle\sigma_{i, z} \sigma_{i+2, z}\right\rangle=-\frac{8}{3} e_{i},
$$

where $e_{i}=\langle H\rangle / N$ denotes the expectation value of the energy corresponding to each site. Especially in this model, no matter what values of the parameters $\theta$ and $\varphi$ are chosen, the EXE $\Delta E_{i}$ gives the same value. Obviously, $\Delta E_{i}$ can be used to characterize the QPTs in the $J_{1}-J_{2}$ model due to the direct relation with the energy level. The energy level crossing point also corresponds to the sudden change of the EXE and thus indicate the QPTs. The relation in Eq. (37) is still true in the excited states, where $\Delta E_{i}$ may present negative values. Therefore, $\Delta E_{i}$ is effective to detect the second critical point $J_{2 c} \approx 0.241$ when taking into account the first excited state energy. Recalling the GMQD of the NN spin pair in Eq. (21), then we have

$$
D_{g}\left(\rho_{12}\right)=\frac{1}{8 J_{1}^{2}}\left(J_{2} \frac{\partial \Delta E_{i}}{\partial J_{2}}-\Delta E_{i}\right)^{2},
$$

and the GMQD of the NNN spin pair

$$
D_{g}\left(\rho_{13}\right)=\frac{1}{8}\left(\frac{\partial \Delta E_{i}}{\partial J_{2}}\right)^{2}
$$



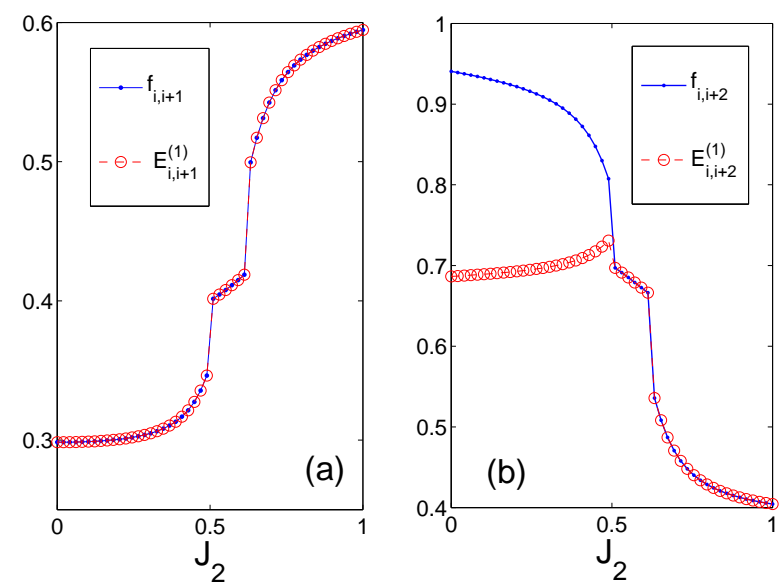

FIG. 5: (a) NN-site frustration measure $f_{i, i+1}$ and its lower bound $E_{i, i+1}^{(1)}$ versus coupling $J_{2}$ for the system size $N=10$; (b) NNN-site frustration measure $f_{i, i+2}$ and its lower bound $E_{i, i+2}^{(1)}$ versus $J_{2}$ for the system size $N=10$. The coupling strength $J_{2}$ is in units of $J_{1}$.

the two equations above connect the pairwise GMQD to the EXE and its derivative. A vanishing EXE will induce a zero pairwise GMQD but the converse may be not true. Finite EXE can also cause vanishing GMQD, because GMQD characterizes the pairwise quantum correlations and not the single-site ones. In other words, frustration can destroy the pairwise correlation and hold the block correlation between single-site and the rest part.

\section{A. Universal measure of frustration and GMQD}

Recently a universal measure of frustration has been introduced as the overlap between the global ground state of a frustrated model and the GS of unfrustrated spin pairs [50]. For a many-body Hamiltonian $H=\sum_{S} h_{S}$ with $h_{S}$ describing the local interaction. The definition of the frustration corresponding to the subsystem $S$ is

$$
f_{S}=1-\operatorname{Tr}\left[\rho \Pi_{S}\right]
$$

where $\rho$ denotes a pure state, and let $\Pi_{S}=|\Psi\rangle_{s}\langle\Psi|$ be the projector onto the subspace $S$ and $|\Psi\rangle_{s}$ is the GS of the local interaction $h_{S}$ which is not frustrated. The quantity $f_{S}$ is a well-defined measure of the interaction $h_{S}$. It turns out that the geometric bipartite groundstate entanglement measured by

$$
E_{S}^{(d)}=1-\sum_{i}^{d} \lambda_{i}^{\downarrow}\left(\rho_{s}\right)
$$

is a universal lower bound to the frustration $f_{S}$, and $d$ is the rank of the projector $\Pi_{S}$. For the degenerate GS $|\Psi\rangle_{s}, d$ indicates the degeneracy. $\lambda_{i}^{\downarrow}$ are the eigenvalues of $\rho_{s}=\operatorname{Tr}_{R}(\rho)$ in decreasing order.
In the $J_{1}-J_{2}$ model, the GS of $h_{S}=J_{1} \mathbf{s}_{i} \cdot \mathbf{s}_{i+1}$ (or $\left.J_{2} \mathbf{s}_{i} \cdot \mathbf{s}_{i+2}\right)$ is the singlet state $\left|\Psi_{-}\right\rangle=(|10\rangle-|01\rangle) / \sqrt{2}$, thus the projector $\Pi_{S}=\left|\Psi_{-}\right\rangle\left\langle\Psi_{-}\right|$with $d=1$. Then we obtain the frustration measure is a function of the correlator as:

$$
f_{i j}=\frac{3}{4}+\frac{3}{4}\left\langle\sigma_{i \alpha} \sigma_{j \alpha}\right\rangle
$$

where $\alpha=x, y, z$, and $j=i+1$ or $i+2$.

From the frustration measure in Eq. (42), we exploit a nonlinear relation with the GMQD that

$$
D_{g}\left(\rho_{i j}\right)=\frac{8}{9}\left(f_{i j}-\frac{3}{4}\right)^{2},
$$

from which, the GMQD achieves the maximum value $1 / 2$ when the frustration vanishes $f_{i j}=0$, and has zero value when a finite frustration exists $f_{i j}=3 / 4$. This implies that the frustration in this model will depress the pairwise correlation, however, it enhances the block correlation between the two-site subsystem and the rest subsystem, which can be verified by calculating the linear entropy

$$
S_{l}\left(\rho_{i j}\right)=1-\operatorname{Tr}\left(\rho_{i j}^{2}\right)=\frac{3}{4}-\frac{4}{3}\left(f_{i j}-\frac{3}{4}\right)^{2},
$$

obviously, when $f_{i j}=3 / 4$, the linear entropy has the maximum value which signals the the maximal bipartite entanglement between the two-site subsystem and the rest part of the system. To note that we now consider the pure GS case, thus the linear entropy is an effective measure of the bipartite entanglement and also the quantum correlation because we also find a direct relation between the linear entropy and the GMQD that

$$
S_{l}\left(\rho_{i j}\right)=\frac{3}{4}-\frac{3}{2} D_{g}\left(\rho_{i j}\right),
$$

from which one can clearly find that the maximal pairwise correlation $D_{g}\left(\rho_{i j}\right)=1 / 2$ will destroy the block correlation between the two-site subsystem and the rest part, then $S_{l}\left(\rho_{i j}\right)=0$.

The total measure of frustration is defined as $F=$ $\frac{1}{M} \sum_{i, j} f_{i j}$, where $M$ is the number of bonds, and in our model $M=2 N$ ( $N$ is the total number of the spin), then we obtain

$$
\begin{aligned}
F & =\frac{1}{2}\left(f_{i, i+1}+f_{i, i+2}\right) \\
& =\frac{3}{4}+\frac{3}{8}\left(\left\langle\sigma_{i, \alpha} \sigma_{i+1, \alpha}\right\rangle+\left\langle\sigma_{i, \alpha} \sigma_{i+2, \alpha}\right\rangle\right) \\
& =\frac{3}{4}+\frac{1}{2 J_{1}}\left[e_{i}+\left(J_{1}-J_{2}\right) \frac{\partial e_{i}}{\partial J_{2}}\right]
\end{aligned}
$$

in the above, we make use of $\left\langle\sigma_{i \alpha} \sigma_{i+1, \alpha}\right\rangle=\frac{4}{3 J_{1}}\left(e_{i}-\right.$ $\left.J_{2} \frac{\partial e_{i}}{\partial J_{2}}\right)$, and $\left\langle\sigma_{i \alpha} \sigma_{i+2, \alpha}\right\rangle=\frac{4}{3} \frac{\partial e_{i}}{\partial J_{2}}$ corresponding to the NN sites and NNN sites respectively. 
Now we calculate the lower bound

$$
E_{i j}^{(1)}=\left\{\begin{array}{l}
\frac{3}{4}+\frac{3}{4}\left\langle\sigma_{i \alpha} \sigma_{j \alpha}\right\rangle \text { when }\left\langle\sigma_{i \alpha} \sigma_{j \alpha}\right\rangle \leq 0, \\
\frac{3}{4}-\frac{1}{4}\left\langle\sigma_{i \alpha} \sigma_{j \alpha}\right\rangle \text { when }\left\langle\sigma_{i \alpha} \sigma_{j \alpha}\right\rangle>0 .
\end{array}\right.
$$

With the help of the energy levels shown in Fig. 1, for the GS energy level, obviously, one can find $\left\langle\sigma_{i \alpha} \sigma_{i+2, \alpha}\right\rangle>0$ when $J_{2}<0.5$, and $\left\langle\sigma_{i \alpha} \sigma_{i+2, \alpha}\right\rangle<0$ when $J_{2} \geq 0.5$. Thus based on the Eq. (47), we have

$$
\left\{\begin{array}{l}
f_{i, i+2}>E_{i, i+2}^{(1)} \text { when } J_{2}<0.5 \\
f_{i, i+2}=E_{i, i+2}^{(1)} \text { when } J_{2} \geq 0.5 .
\end{array}\right.
$$

It is known that the inequality $f_{i j}>E_{i j}^{d}$ implies the appearance of the geometric frustration [50]. Hence, we find that the geometric frustration appears when $J_{2}<0.5$ and vanishes when when $J_{2} \geq 0.5$. It implies that the frustration measure and its lower bound can reflect the change of the fundamental structure of the system, such as the energy level crossing, therefore, they can be used to indicate the first-order QPT point $J_{c 1}=0.5$.

Based on the analytical results of the GS energy level in the 4-site and 6-site case, we calculate the frustration measure. For 4 -site case (let $J_{1}=1$ ): the frustration of the NN sites

$$
f_{i, i+1}=\left\{\begin{array}{l}
\frac{1}{4}=E_{i, i+1}^{(1)} \text { when } J_{2}<0.5, \\
\frac{3}{4}=E_{i, i+1}^{(1)} \text { when } J_{2} \geq 0.5 .
\end{array}\right.
$$

and the frustration of the NNN sites

$$
f_{i, i+2}=\left\{\begin{array}{c}
1>E_{i, i+2}^{(1)}=\frac{2}{3} \text { when } J_{2}<0.5, \\
0=E_{i, i+2}^{(1)} \text { when } J_{2} \geq 0.5 .
\end{array}\right.
$$

For 6-site case, we have the frustration of the NN sites

$$
f_{i, i+1}=\left\{\begin{array}{c}
\frac{9 J_{2}-13+7 \Omega}{12 \Omega}=E_{i, i+1}^{(1)} \text { when } J_{2}<0.5 \\
\frac{1}{2}=E_{i, i+1}^{(1)} \text { when } J_{2} \geq 0.5
\end{array}\right.
$$

where $\Omega=\sqrt{9 J_{2}^{2}-18 J_{2}+13}$, and the frustration of the NNN sites

$f_{i, i+2}=\left\{\begin{array}{c}\frac{3\left(\Omega-J_{2}+1\right)}{4 \Omega}>E_{i, i+2}^{(1)}=\frac{3 \Omega+J_{2}-1}{4 \Omega} \text { when } J_{2}<0.5, \\ \frac{1}{2}=E_{i, i+2}^{(1)} \text { when } J_{2} \geq 0.5 .\end{array}\right.$

For larger size system such as $N=10$, we numerically calculate the frustration measure and its lower bound in Fig. 5. Both the NN-site frustration measure $f_{i, i+1}$ in subfigure (a) and the NNN-site frustration measure $f_{i, i+2}$ in subfigure (b) present a sudden change at the QPT point $J_{2}=0.5$. Moreover, in the subfigure (a), we shows that the NN-site frustration measure $f_{i, i+1}$ and its lower bound $E_{i, i+1}^{(1)}$ are consistent with each other, which implies that only quantum frustrations exist between NN sites. Differently, in the subfigure (b), we find the NNNsite frustration $f_{i, i+2}>E_{i, i+2}^{(1)}$ when $J_{2}<0.5$, which signals the appearance of the geometric frustration.

From the above, we find the nonlinear dependence of the GMQD on the frustration, which implies that the pairwise quantum correlation characterized by the GMQD is greatly affected by the frustration, e.g., some finite frustration as $f_{i j}=3 / 4$ can depress the GMQD to zero. On the other hand, we believe that the frustration measure can be used to characterize the first-order QPT in the $J_{1}-J_{2}$ model and also other systems. In addition, the inequality $f_{S} \geq E_{S}^{(d)}$ holds as well in any mixed states [50], thus the method is also effective to detect the first-order QPTs in the degenerate GSs. In our $J_{1^{-}} J_{2}$ model, the quantity $f_{S}$ can also be used in the first-excited states to detect the QPT point $J_{c 2} \approx 0.241$. However, in the excited states (ESs) case, the quantity $f_{S}$ can not be understood as the frustration measure, instead, it only quantifies how much fails to fully overlap with the subspace selected by the projector $\Pi_{S}$, and the projector $\Pi_{S}$ may come from the GS or the ES of the local interaction $h_{S}$.

\section{CONCLUSION}

In this paper, we considered the quantum discord (QD) in the Heisenberg spin chain with next-nearest-neighbor (NNN) interaction. By using the geometric measure of the quantum discord (GMQD), we studied the quantum correlation properties of the ground states (GSs) and the first excited states (ESs).

We give a general analytical result of the GMQD for the $X$-type states. For the Heisenberg system with the $S U(2)$ symmetry and $Z_{2}$ symmetry, we give an exact relation between the GMQD and the two-site correlators. Furthermore, the connection between GMQD and the eigenenergies was revealed. For the 4 -site and 6-site cases, the analytical results of the GMQD for the GS and first ES are obtained, from which the first critical point $J_{c 1}=0.5$ can be exactly detected. We also numerically studied the NN-site and NNN-site GMQD of the ESs. It is found that when the system size increases from 6 sites to 10 sites, the discontinuous point of the GMQD tends to the second QPT point $J_{c 2} \simeq 0.241$.

Moreover, by using the entanglement excitation energy and a universal frustration measure we considered the frustration properties of the system and find the nonlinear dependence of the GMQD on the frustration. The measure of the frustration can also be employed to detect the QPTs in this system.

We emphasize that the two-site GMQD can detect the QPTs in this Heisenberg system with NNN interaction. Although the two-site GMQD approach is only effective in the finite-size systems, it has practical significance for the usual theoretical and experimental studies. The problems of the GMQD (or QD) in other systems with QPTs are interesting and need further consideration. 


\section{Acknowledgements}

This work was supported by the National Nature Science Foundation of China with Grant No.11005027; the Natural Science Foundation of Zhejiang Province with Grant No. Y6090058; the Program for HNUEYT with Grant No. 2011-01-011; the NFRPC through Grant
No. 2012CB921602 and the NSFC through Grants No. 11025527 and No. 10935010.

\section{References}

[1] H. Ollivier and W. H. Zurek, Phys. Rev. Lett. 88, 017901 (2001).

[2] L. Henderson and V. Vedral, J. Phys. A 34, 6899 (2001); V. Vedral, Phys. Rev. Lett. 90, 050401 (2003).

[3] S. Luo, Phys. Rev. A 77 , 042303 (2008).

[4] B. Liu, B. Shao and J. Zou, Phys. Rev. A 82, 062119 (2010).

[5] A. Datta and G. Vidal, Phys. Rev. A 75, 042310 (2007).

[6] K. Modi, T. Paterek, W. Son, V. Vedral, and M. Williamson, Phys. Rev. Lett. 104, 080501 (2010).

[7] X. Hu, Y. Gu, Q. Gong, and G. Guo, Phys. Rev. A 84, 022113 (2011).

[8] Q. He, J. Xu, D. Yao, and Y. Zhang, Phys. Rev. A 84, 022312 (2011).

[9] L. Wu and D. Segal, Phys. Rev. A 84, 012319 (2011).

[10] L. Cen, X. Li, J. Shao, and Y. Yan , Phys. Rev. A 83, 054101 (2011).

[11] B. Li, Z. Wang, and S. Fei , Phys. Rev. A 83, 022321 (2011).

[12] Z. Li, J. Jin, and C. Yu, Phys. Rev. A 83, 012317 (2011).

[13] B. Daki, V. Vedral, and C. Brukner, Phys. Rev. Lett. 105, 190502 (2010).

[14] C. C. Rulli and M. S. Sarandy, Phys. Rev. A 84, 042109 (2011).

[15] Q. Chen, C. Zhang, S. Yu, X.X.Yi, and C. H. Oh, Phys. Rev. A 84, 042313 (2011).

[16] M. Ali, A. R. P. Rau, and G. Alber, Phys. Rev. A 81, 042105 (2010).

[17] L. Ciliberti, R. Rossignoli, and N. Canosa, Phys. Rev. A 82, 042316 (2010).

[18] D. Cavalcanti, L. Aolita, S. Boixo, K. Modi, M. Piani, and A. Winter, Phys. Rev. A 83, 032324 (2011).

[19] D. Girolami and G. Adesso, Phys. Rev. A 83, 052108 (2011).

[20] Q. Chen, C. Zhang, S. Yu, X.X. Yi, and C.H. Oh, Phys. Rev. A 84, 042313(2011).

[21] B. Dakić, V. Vedral, and Č. Brukner, Phys. Rev. Lett. 105, 190502 (2010).

[22] S. Gu, S. Deng, Y. Li, and H. Lin, Phys. Rev. Lett. 93, 086402 (2004).

[23] Román Orús, Phys. Rev. Lett. 100, 130502 (2008).

[24] Y. Chen, P. Zanardi, Z. D. Wang, and F. C. Zhang, New J. of Phys. 897 (2006).

[25] Z. Sun, X. Wang, and C. P. Sun, Phys. Rev. A 75, 062312 (2007).
[26] L. A. Wu, M. S. Sarandy, and D. A. Lidar, Phys. Rev. Lett. 93, 250404 (2004).

[27] S. Zheng, Phys. Rev. A 84, 033817 (2011).

[28] F. Ma, S. Liu, and X. Kong, Phys. Rev. A 84, 042302 (2011).

[29] H. Xiong, J. Ma, Z. Sun, X. Wang, Phys. Rev. B 79, 174425 (2009);H. Xiong, J. Ma, Y. Wang and X. Wang, J. Phys. A: Math. Theor. 42, 065304 (2009).

[30] T. Werlang, C. Trippe, G. A. P. Ribeiro, and G. Rigolin, Phys. Rev. Lett. 105 105, 095702 (2010).

[31] S. Gu, H. Kwok, W. Ning, and H. Lin, Phys. Rev. B 77, 245109 (2008).

[32] Z. Sun, X. Lu, H. Xiong and J. Ma, New J. Phys 11 113005 (2009).

[33] S. Chen, L. Wang, S. Gu, and Y. Wang, Phys. Rev. E 76, 061108 (2007).

[34] Z. Sun, Phys. Rev. A 84, 052307 (2011).

[35] T. Werlang, G. A. P. Ribeiro, and G. Rigolin, Phys. Rev. A 83, 062334 (2011).

[36] Z. Sun, X. Lu and L. Song, J. Phys. B: At. Mol. Opt. Phys. 43, 215504 (2010).

[37] B. Liu, B. Shao, and J. Zou, Phys. Rev. A 82, 062119 (2010).

[38] M. S. Sarandy, Phys. Rev. A 80, 022108 (2009).

[39] M. Hase, I. Terasaki, and K. Uchinokura, Phys. Rev. Lett. 703651 (1993).

[40] C. K. Majumdar and D. K. Ghosh, J. Math. Phys. 10, 1388 (1969); J. Phys. C 3, 911 (1970).

[41] F. D. M. Haldane, Phys. Rev. B 25, 4925 (1982).

[42] B. Groisman, S. Popescu, and A. Winter, Phys. Rev. A 72, 032317 (2005).

[43] J. Schlienz and G. Mahler, Phys. Rev. A 52, 4396 (1995).

[44] X. Lu, Z. Xi, Z. Sun and X. Wang, Quantum Information \& Computation 10 (2010) 11\&12.

[45] H. Q. Lin, Phys. Rev. B 42, 6561 (1990).

[46] X. G. Wang, Physics Letters A 329439 (2004).

[47] Z. Sun, X. Wang, A. Hu, and Y. Li, Commun. Theor.Phys 43 1033-1036 (2005).

[48] S. M. Giampaolo, G. Adesso, and F. Illuminati, Phys. Rev. Lett. 104, 207202 (2010).

[49] S. M. Giampaolo and F. Illuminati, Phys. Rev. A 76, 042301 (2007);

[50] S. M. Giampaolo, G. Gualdi, A. Monras, and F. Illuminati, Phys. Rev. Lett. 107, 260602 (2011). 